\title{
AN INTEGRATED RELAP5-3D AND MULTIPHASE CFD CODE SYSTEM UTILIZING A SEMI-IMPLICIT COUPLING TECHNIQUE
}

\author{
D. L. Aumiller, E. T. Tomlinson \\ Bettis Atomic Power Laboratory \\ W. L. Weaver \\ Idaho National Engineering and Environmental Laboratory
}

DE-AC11-98PN38206

\section{NOTICE}

This report was prepared as an account of work sponsored by the United States Government. Neither the United States, nor the United States Department of Energy, nor any of their employees, nor any of their contractors, subcontractors, or their employees, makes any warranty, express or implied, or assumes any legal liability or responsibility for the accuracy, completeness or usefulness of any information, apparatus, product or process disclosed, or represents that its use would not infringe privately owned rights. 


\title{
An Integrated RELAP5-3D and Multiphase CFD Code System Utilizing a
}

\section{Semi-Implicit Coupling Technique}

\author{
D.L. Aumiller ${ }^{a}$, E.T. Tomlinson ${ }^{a}$, W. L. Weaver ${ }^{b}$ \\ ${ }^{a}$ Bettis Atomic Power Laboratory, P.O. Box 79, West Mifflin, PA 15122-0079, USA \\ ${ }^{b}$ Idaho National Engineering and Environmental Laboratory, P.O. Box 1625, Idaho Falls, ID 83415, USA
}

\begin{abstract}
An integrated code system consisting of RELAP5-3D and a multiphase CFD program has been created through the use of a generic semi-implicit coupling algorithm. Unlike previous CFD coupling work, this coupling scheme is numerically stable provided the material Courant limit is not violated in RELAP5-3D or at the coupling locations. The basis for the coupling scheme and details regarding the unique features associated with the application of this technique to a four-field CFD program are presented. Finally, the results of a verification problem are presented. The coupled code system is shown to yield accurate and numerically stable results.
\end{abstract}

\section{Introduction}

One of the challenging problems in safety analysis has always been providing the desired degree of physical modeling without burdening the user with the need for excessive computational resources. In the past, individual thermal-hydraulic programs have attempted to provide a balance between complexity and runtime/flexibility that was appropriate for a particular class of problems. The result is a spectrum of programs that range from the drift-flux based RETRAN program (Paulsen, 1996) which is used to analyze plant transients, to the two-fluid RELAP5-3D (RELAP5-3D Development Team, 1999) and TRAC-PF1 (Schnurr, 1992) programs which are used to analyze loss-ofcoolant accidents, to the three-field COBRA-TF program which has been used to model reflood heat transfer (Paik, 1985).

The most detailed of the current thermal-hydraulic programs are the multi-phase Computational Fluid Dynamics (CFD) programs. These programs are capable of calculating detailed flow field predictions through the use of more detailed physical models. Heretofore, they have not been utilized for safety analyses for two primary reasons: the general lack of multi-phase CFD programs and the exorbitant run time usually associated with these programs. Recent work in the area of multi- phase CFD and the advent of ever faster computers has made it feasible to perform some CFD based calculations in the context of a safety analysis.

Ideally, an analyst would be able to choose an appropriate level of modeling detail in various regions of a simulation based on the modeling requirements as opposed to having the requirements dictated by the choice of a particular computer program. This idea has led to the coupling of various computer programs to provide this capability. The best known example of this is the COBRA/TRAC program (Thurgood, 1983).

More recently, Aumiller et al. (2001) coupled RELAP5-3D to a CFD program using a simplistic explicit numerical technique. The work described by Aumiller, which included an analysis of the Edwards and O'Brien (1970) blowdown problem, showed the feasibility of using a coupled RELAP5-3D/CFD code system to perform two-phase fast transient calculations. However, numerical instabilities were observed in the calculation and were attributed to the explicit coupling algorithm.

To eliminate the numerical instabilities associated with explicit numerics, Weaver et al. (2000) developed a new generic semi-implicit coupling technique. Weaver provides a good discussion concerning the numerical 
stability issues related to thermal-hydraulic code coupling. A brief discussion of the methodology is presented later in this paper.

The most important features of the semi-implicit coupling methodology are its generality and its use of the Parallel Virtual Machine (PVM) software package (Geist, 1994) to allow communication among the programs. These features allow additional programs to be added to the integrated code system in a very straight forward manner. Additionally, by creating this generic interface, the development of all of the programs in the integrated system can proceed independently of each other, provided the interface is maintained. This approach is contrasted with the "hard-wired" approach used in COBRA/TRAC where the programs are conjoined to form one new program. This approach makes code maintenance and development more difficult. This may partially explain why the COBRA/TRAC program has not kept current with the development of either the COBRA or TRAC programs.

\section{Semi-Implicit Coupling Algorithm}

A complete description of the semi-implicit coupling algorithm is provided by Weaver (2000); what follows is a brief synopsis of the methodology. A coupled code system performs a domain decomposition of the complete problem to allow each program to solve a piece of the problem. This is shown schematically in Figure 1 . The advantage of the semi-implicit coupling technique is that it is more numerically stable than simpler, explicit coupling schemes.

The use of implicit velocities and pressures in the discretized conservation equations in the semi-implicit numerical method (Liles, 1978) provides numerical stability for time step sizes smaller than the material Courant limit. One feature of this method is that a single matrix containing only new-time pressures can be developed. This matrix contains the effect of all of the new-time variables. This feature is the key to the semiimplicit coupling algorithm.

Using the nomenclature of Weaver (2000), RELAP5-3D will be the master process and the CFD program will be the slave process in this system. The semi-implicit coupling methodology modifies the solution procedure in the RELAP5-3D program for the junctions representing the connections between the two systems. The pressure equation for the volume attached to the coupling location in the RELAP5-3D computational domain is modified by retaining the mass, energy, volume and non-condensable gas flow rates as unknowns.

By retaining these terms, the changes in the pressures in all of the volumes in the computational domain can be computed in terms of the flow rates in the coupling junctions as

$$
\begin{aligned}
\delta P_{k}^{n+1}= & a_{k}+\sum_{j=1}^{N_{c}} b_{k, j} n_{g, j}^{n+1}+ \\
& \sum_{j=1}^{N_{c}} c_{k, j} u_{g, j}^{n+1}+\sum_{j=1}^{N_{c}} d_{k, j} u_{f, j}^{n+1}+ \\
& \sum_{j=1}^{N_{c}} e_{k, j} m_{g, j}^{n+1}+\sum_{j=1}^{N_{c}} f_{k, j} m_{f, j}^{n+1}+ \\
& \sum_{j=1}^{N_{c}} g_{k, j} w_{g, j}^{n+1}+\sum_{j=1}^{N_{c}} h_{k, j} w_{f, j}^{n+1}
\end{aligned}
$$

where $n_{g}, u_{g}, u_{1}, m_{g}, m_{l}, w_{g}$ and $w_{1}$ represent the flow rate of non-condensable gas, and the phasic flow rates of energy, mass and volume at the coupling locations, and $\mathbf{N}_{\mathrm{c}}$ is the number of coupling junctions. The coefficients a through $\mathbf{h}$ for the volumes attached to the coupling junctions in the RELAP5-3D computational domain (volumes 1 and 2 in Figure 1) are then transmitted to the CFD program. The CFD solution uses coefficients a through $\mathbf{h}$ to calculate the interdependence of pressure and flow rates consistent with RELAP5-3D solution strategy. This consistency is the key to the semi-implicit coupling methodology. When the mass, energy, volume and non-condensable flow rates in the coupling junctions have been received from the slave process, Equation (1) can be evaluated for the change in the pressure in each volume in the RELAP5-3D system. Once the changes in the pressures in the volumes have been computed, the time advancement may be completed in the normal manner.

The semi-implicit coupling algorithm is numerically stable subject to certain limitations. The master process (RELAP5-3D in this application) must use semiimplicit numerics and the material Courant limit must not be violated in the master process or at the coupling location. The semi-implicit coupling algorithm does not influence the stability of the slave process (the CFD program in this application). At this point, a few words concerning stability of the CFD program are appropriate. The CFD program which was chosen for this work uses a fully-implicit solution procedure. Through the use of fully-implicit numerics, the CFD 
program has no formal time step size requirements for numerical stability. This is not to say that the iterative solution procedure will converge for any size time step; it will not. However, since the material Courant limit does not determine stability, the nodalization of the CFD program can be small enough to resolve the fluid flow patterns in a manner typical for CFD calculations without violating any formal stability criteria.

It should be noted that the semi-implicit coupling algorithm can be implemented as a master process for any number of system codes. However, the implementation into the RELAP series of codes is easier since they use a "single-shot" linearization technique. By only linearizing the conservation equations once per time step, the coupling coefficients remain fixed during the course of the time step. If the conservation equations are linearized more than once per time step, new coupling coefficients would be calculated at each iteration in the master process and the slave process would need to recalculate the flow field for each new set of coupling coefficients. In the present implementation in the CFD program, this additional requirement would result in significantly longer execution times.

\section{Implementation in the CFD Program}

The CFD program which was chosen to be coupled with RELAP5-3D was based on the CFDS-FLOW3D (Harwell Laboratory, 1992) (now CFX) program. The program has been extensively modified to provide multidimensional, multifield, heated, two-phase flow capability. A four-field formulation [continuous liquid, dispersed vapor (bubbles), continuous vapor and dispersed liquid (drops)] is used to represent the complete range of two-phase flow patterns from bubbly through annular flow more accurately.

As stated previously, the role of the CFD program in this coupling algorithm is to calculate the phasic flow rates of mass, energy, volume and gaseous non-condensables across the coupling plane. (For the remainder of this paper, the phrase "net phasic flows rate" will refer to the net phasic flow rates of mass, energy, volume, and the mass flow rate of a non-condensable gas). Using the CFD program to calculate the net phasic flow rates across the coupling plane instead of calculating volume conditions has many advantages. The first of these is the ability to integrate the CFD results over the flow area at the coupling plane. Since the coupling algorithm is a function of only the net phasic flow rates, this technique readily permits the coupling of one RELAP5-3D volume to numerous CFD volumes. This is a requirement of any coupled system/CFD code suite, since the nodalization of system programs, such as RELAP5-3D, is usually much coarser than the nodalization used for the CFD programs.

The semi-implicit coupling in the CFD program is implemented as an extension of a standard pressure boundary condition. At the beginning of each time step, RELAP5-3D passes the old-time volume parameters (pressure, void fraction, phasic densities, phasic internal energies and non-condensable quality) to the CFD program. Using these conditions, the CFD program then performs the spatial differencing (upwind differencing was used in this example) of the quantities convected across the boundary (void fraction, phasic densities, phasic internal energies, phasic velocities and noncondensable quality). Since the CFD program may use many more cells and a larger number of fields to represent the fluid conditions, an averaging scheme is required to define the two-phase state variables required by RELAP5-3D. In the current implementation, the upwind quantity for each of the CFD cells is computed as a simple volume weighted average. Note that this implementation will correctly handle counter-current phasic flow situations since each small cell is individually examined.

At this point in the solution scheme, the convected quantities are fixed for the time step. Using these convected quantities, RELAP5-3D creates the pressure matrix as described above and transmits coefficients a through $h$ to the CFD program. The CFD program uses these coefficients in conjunction with the net phasic flow rates at the coupling plane. These net phasic flow rates are calculated using

$$
\text { Net Phasic Flow }=\sum_{i=1}^{\text {nfld nfac }} \sum_{j=1} A_{j} v_{i, j} \phi_{i, j}
$$

where nfld, is the number of fields that are present for the given phase, and nfac is the number of faces in the CFD program that comprise the coupling junction, $A_{j}$ is the flow area for the face, $V_{i, j}$ is the velocity and $\phi_{i, j}$ is the convected quantity (e.g., macroscopic density for the mass equation). This has been implemented in such a way as to maintain the use of symmetry boundary conditions in the CFD program by using a multiplier (1, 2 or 4 ) depending on how many symmetry planes are used in the problem.

Note that this definition integrates over the number of fields in a given phase. This allows the CFD program to calculate counter-current phasic flows (i.e., a falling liquid film and rising liquid drops) at the coupling plane and determine the net phasic flow rates. 
If a direct solver was available in the CFD program, it would be possible to incorporate the coupling coefficients directly into the solution procedure; however, the CFD program uses an iterative technique and therefore the coupling effects must also be calculated iteratively. This process involves the calculation of all of the net phasic flow rates at each iteration. The pressures in the coupling volumes are then updated based on these new net phasic flow rates and the iterative solution continues. The current implementation underrelaxes the update of the pressures in the coupling volume. Since the boundary conditions are now updated in the iterative procedure, a check must be performed to ensure that the boundary conditions have converged. In the current implementation, the relative change in any of the net phasic flow rates must not exceed $10^{-4}$ between iterations. Once a converged CFD solution has been obtained, the net phasic flow rates are sent to RELAP5-3D which uses them to calculate the new-time pressures in the coupling volumes. These pressures are then used in the back-substitution process to calculate the remaining RELAP5-3D new-time variables. This completes one time step and the process is repeated for the next time step.

In addition to collapsing the fields at the coupling plane to determine the net phasic flow rates, the coupling algorithm must also create data to translate from the two-field volume conditions represented by the RELAP5-3D program to the four-field representation used by the CFD program. In the current implementation, data creation is done through the use of a function to determine the fraction of the phase, liquid or gas, that is in the dispersed form. Physically, it is expected that in the limit that there is no liquid or gas, all of what is present would be in the dispersed phase as either a few drops or a few small bubbles. Therefore, the functions should have this feature. Furthermore, the functions should be continuous in the first derivative at the extremes where either of the fields disappears. A generic function to represent the transition from dispersed to continuous has been defined as

$\frac{\alpha_{\text {disp }}}{\alpha_{\text {total }}}=\frac{1}{2}\left(1+\frac{2}{\pi} \operatorname{atan}\left(y\left(\alpha_{\text {total }}\right)\right)\right)$

where the function $y\left(\alpha_{\text {total }}\right)$ is given by

$$
\begin{aligned}
y\left(\alpha_{\text {total }}\right)= & -\left|\frac{1}{\tilde{y}_{2}\left(\alpha_{\text {total }}\right)}\right|^{2 n+1} \\
& +\left(\frac{1}{\tilde{y}_{2}\left(\alpha_{\text {total }}\right)}\right)^{2 n+1} \\
& +n \tilde{y}_{2}\left(\alpha_{\text {total }}\right)
\end{aligned}
$$

where the intermediate function $\tilde{y}_{2}\left(\alpha_{\text {total }}\right)$ is

$$
\begin{aligned}
& \tilde{y}_{2}(x)=\max \left(-1,\left(\min \left(1, \tilde{y}_{1}(x)\right)\right)\right) \\
& \tilde{y}_{1}(x)=-1+2 \frac{x-x_{\min }}{x_{\max }-x_{\min }}
\end{aligned}
$$

The values used for the different phases are given in Table 1. These curves are shown in Figure 2. In the future, information regarding the flow regime in the coupled cell could be used to provide a more mechanistically based procedure to apportion the phases between their dispersed and continuous components.

Table 1: Parameters Used in Phase Splitting

\begin{tabular}{|c|c|c|c|}
\hline Phase & $\mathrm{x}_{\min }$ & $\mathrm{x}_{\max }$ & $\mathrm{n}$ \\
\hline Gas & 0.4 & 0.9 & 0.5 \\
\hline Liquid & 0.0 & 0.6 & 1.0 \\
\hline
\end{tabular}

The numbers used for the limits and the slope have been chosen to approximate the transition from bubbly flow to annular flow. The choice of $x_{\min }$ for the gas phase and $x_{\max }$ for the liquid phase represent an assumption that a bubbly flow regime will exist for all void fractions less than $40 \%$. The choice of $x_{\max }$ for the gas phase represents the void fraction above which annular flow is expected to occur. Finally, the choice of 0.0 for the liquid phase $x_{\min }$ indicates that a thin liquid film could coexist with droplets if any liquid is present. It should be noted, that these parameters and this technique is not considered to be optimal for all situations and current work is ongoing to define a more mechanistic approach to split a two-field representation into a four-fields.

Finally, it should be noted that all of the distributions in the pressure boundary conditions are assumed to be uniform. For some flow regimes (i.e. annular or horizontally stratified flow) this is not appropriate; however, this has not had any adverse impact on any calculations to date. Future work will focus on providing a better solution to this issue.

For this implementation, mass and energy are conserved; however, momentum is not conserved. This is because both RELAP5-3D and the CFD code do not have all of the required information to correctly calculate the $V \nabla V$ term at the pressure boundary locations. In this implementation, the $V \nabla V$ term is set to zero at the coupling planes. This simplification will most adversely effect the accuracy if there is a significant axial velocity gradient at a coupling location. 
The error associated with the non-conservation of momentum is deemed to be small for the verification problem described later because no such large axial velocity gradients exist at the coupling locations. A judicious choice of coupling locations should mitigate the effect of this issue until methods for conserving momentum are developed and implemented in the coupling algorithm.

\section{Programming Improvements to the RELAP5-3D Implementation}

As described by Weaver (2000), there are several drawbacks to the implementation of the semi-implicit coupling technique in RELAP5-3D. These limitations make the implementation described by Weaver (2000) more academic than useful for real world problems. This section will describe the solution of these issues which make the technique viable for real problems.

The most restrictive limitation in the previous implementation is the inability to handle a code failure for a given time step. All thermal-hydraulic programs provide a determination of the validity of a given solution. If the solution is determined to be unacceptable, the solution procedure returns to the previous solution and usually proceeds with a smaller time step size. This process is typically referred to as a backup. In the previous implementation, if either program required a backup, the solution was aborted. This has been remedied by the inclusion of a message from both programs to the other to signal whether or not a good solution has been obtained. If either program requests a backup, both programs perform the backup and proceed with a new time step size.

This leads directly to the next issue. In the previous implementation, both programs were required to independently know the proper time step size as a function of time. There was no mechanism for exchanging time step size information between the programs. In the current implementation, the timestep size will be decreased if either program requests a reduction; however, the timestep size can only be increased if both programs agree.

Another issue that has been addressed in this implementation is the simultaneous writing of restart and output files. Since both programs have a restart capability, it is advantageous to preserve this capability for the integrated code system. However, for this feature to work, both programs must write the files at the same time. This has been accomplished by using the mechanism already in RELAP5-3D to determine the timing of writing of these files. Using this approach, whenever RELAP5-3D writes to the restart or output file, a message is sent to the CFD program so that it can do the same.

The final programming improvement that has been made is the ability for RELAP5-3D to pass a normalized power variable to the CFD program. This was done to take advantage of RELAP5-3D's many different ways of calculating a transient power history that are not native to the CFD program. For example, RELAP5-3D can perform point or nodal kinetics calculations or use a table look-up function to determine a transient power, while the CFD program contains neither of these features.

\section{Verification Testing}

A test case was developed to verify the implementation of the semi-implicit coupling algorithm in the RELAP5-3D/CFD integrated code system. The previous work by Weaver $(2000)$, verified that the semi-implicit coupling had been correctly implemented in RELAP5-3D. This study uses a similar problem and master RELAP5-3D input. Since, the RELAP5-3D implementation is known to work. This section will concentrate on the implementation details for the CFD program.

There are two versions of the test case. In the first version of the test case the entire system is simulated as a single RELAP5-3D problem. The second version of the test case divides the test system into two parts which are simulated as a coupled problem using the semiimplicit coupling methodology. Figure 3 is a schematic coupled problem.

The test case is based on Run 15 of the Christensen (1961) subcooled boiling experiments. Unlike the experiments, the input model includes a parallel flow path for purposes of testing the coupling methodology and uses hydraulic resistances to remove the characteristic oscillations. To provide a transient problem, the pressure difference linearly increases between 0 and 2 seconds. The problem is unheated for the first 5 seconds at which time the power is linearly increased to its maximum value at 10 seconds. This increase in power is achieved through a table look-up in RELAP5-3D, which normalizes the power and transmits the normalized power value to the CFD program for its use. 
In the full calculation, the test section is modeled by a single heated pipe component and the bypass is modeled as an unheated pipe component. The two parallel flow paths are connected to common single volumes at the top and bottom of the test section. These volumes are in turn connected to time dependent volumes. These time dependent volumes impose a pressure gradient on the system. This calculation has been included to provide a qualitative assessment of the coupled code and not to provide a quantitative benchmark. It is fully expected that the differing degrees of modeling complexity will yield different results for the coupled and full problems. The magnitude of these differences will be compounded by the use of parallel flow paths and a pressure forced problem which will cause different total and test section mass flow rates. These different flow rates will in turn cause different void fraction profiles.

The coupled simulation is constructed from two input files. The first input file, for the RELAP5-3D domain, contains the upper and lower common volumes, the time dependent volumes, the bypass channel and the upper portion of the test section. The lowest 15 volumes of the test section were removed and moved to the CFD domain. Coupling volumes and coupling junctions were added to each input file as appropriate. Dotted lines in Figure 3 indicate data exchanges between the coupling volumes and coupling junctions. Boundary volumes in the master system are shown with dotted outlines because they do not contribute boundary conditions to the solution but are required by the input checker in RELAP5-3D.

The nodalization in the CFD portion is finer than in the RELAP5-3D domain. A total of 30 axial mesh points are used, compared with 15 in the RELAP5-3D input. However, the CFD program uses a two-dimensional mesh, with 10 cells the direction normal to the heated surface. A symmetry plane is used in the transverse direction to provide an equivalent of 20 mesh points. The use of a two-dimensional CFD program will exercise the integration requirements to determine the proper net phasic flow rates. This problem was run as a four-field problem to examine the issues of data creation and integration at the coupling locations.

When compared to the nodalization used by Weaver (2000) to verify the RELAP5-3D to RELAP5-3D semiimplicit coupling, the coupling location has been moved. In the problem described by Weaver, the bottom coupling location is located one-quarter of the way up the test section; however, the problem described in this paper places it at the bottom of the test section. The reason for making this choice is that the location used by Weaver is inappropriate for use in this work because transverse profiles are expected at that location. Physically, subcooled boiling is characterized by a wall peaked void fraction profile. In the RELAP5-3D problem the void is considered to be distributed uniformly, but the CFD program can resolve this profile, as will be shown later.

The issue of where the programs are coupled can be an important aspect directly impacting the accuracy of the final solution. As previously stated, this implementation assumes uniform distributions of the RELAP5-3D parameters such as temperature and void fraction in the CFD boundary conditions. If this assumption is incorrect, the use of these inappropriate set of boundary conditions will result in a loss accuracy in the CFD program. Therefore, when coupling a CFD program to a program that uses coarser nodalization, the coupling location should be at a location where the flow field is expected to be uniform to avoid this problem.

Since the CFD program will calculate a different pressure drop and axial void fraction distribution than RELAP5-3D, the results between the full RELAP5-3D solution and the coupled solution can not be used to verify that the implementation has been performed correctly. The essence of the semi-implicit coupling methodology is that the two different programs must use the same relationship between new-time flow rates and pressures. Therefore, the new-time pressure predictions between the master and slave processes for the coupling volumes must be compared. While implementing the methodology, these numbers were often compared and were shown to be correct to machine precision.

Figure 4 shows the results of a comparison of the pressure in the lower coupling volume. For the coupled problem, the RELAP5-3D and CFD solutions are identical to the precision printed in the data files.

Figures 5 shows the predicted mass flow rates at both of the coupling planes. It can be seen that the coupled code conserves mass. It is interesting to note that the pressure drop calculation in the CFD program yields a larger mass flow rate in the coupled analysis when compared to the full RELAP5-3D calculation.

One of the important implementation issues is the correct integration over the number of fields and over the flow area to determine the correct net phasic flow rates. Figure 6 shows the profiles predicted by the CFD program for both the dispersed and continuous vapor fields at the exit of the CFD domain at 60 seconds. This plot clearly shows both a strong spatial variation and 
quite different profiles for the two fields. Given the fact that the mass and energy are conserved, and the pressures are calculated to be identical means that the integration over both the number of fields and the flow area is correct.

The final calculation to be verified is the determination of the proper convected quantities at the coupling planes. Figure 7 is a plot of the convected void fraction at the upper coupling location. When the profiles shown in Figure 6 are volume weighted, the proper convected void fraction at 60 seconds is calculated.

\section{Conclusions}

An integrated code system utilizing RELAP5-3D and a multiphase CFD program has been developed. A generic and numerically stable coupling algorithm has been implemented in the CFD program to eliminate weaknesses that have been seen in previous CFD coupling work.

The particular implementation described in this paper has been shown to be particularly well suited for use in the CFD program. Specifically, the use of the CFD program to calculate the net phasic flow rates at the coupling boundaries provides for a simple integration scheme to convert from a four-field fine nodalization CFD program to a two-field, coarse nodalization safety code.

Implementation details for the CFD program and programming improvements in the semi-implicit coupling algorithm have been discussed in detail. This discussion shows the viability of the methodology for real-world applications.

The results of the test problem indicate that the coupling algorithm has been correctly implemented in both the CFD program and in RELAP5-3D. The ability to integrate CFD and system codes in a generic and numerically stable manner provides a much wider range of flexibility and computational power than exists in current standalone thermal-hydraulic safety codes.

\section{References}

Aumiller, D.L., Tomlinson, E.T., Bauer, R.C., 2001, “A Coupled RELAP5-3D/CFD methodology with a proofof-principle calculation," Nucl. Eng.and Design, 205, 83-90.
Christensen H., 1961. "Power-to-void transfer functions," ANL-6385, AEC Research and Development Report.

Edwards, A.R., O'Brien T.P., 1970. "Studies of phenomena connected with the depressurization of water reactors," Journ. of the Brit. Nucl. Ener. Soc. 9, 125-135.

Geist, A., et al., 1994. "PVM3 user's guide and reference" ORNL/TM-12187, Oak Ridge National Laboratory.

Harwell Laboratory, 1992. FLOW3D release 3.2: user manual, AEA Industrial Technology, Oxfordshire, United Kingdom.

Liles, D.R., Reed W.H., 1978. "A semi-implicit method for two-phase fluid dynamics," Journ. of Comp. Phys. $26,390-407$.

Paik, C., Hochreiter, L., Kelly, J., Kohrt, R., 1985. Analysis of FLECHT-SEASET 163 - rod blocked bundle data using COBRA-TF, NUREG/CR-4166.

Paulsen, M., et al., 1996. RETRAN-3D a program for transient thermal-hydraulic analysis of complex fluid systems, EPRI NP-7450.

RELAP5-3D Development Team, 1999. RELAP5-3D code manuals, Volumes I, II, IV, and V, Idaho National Engineering and Environmental Laboratory, INEELEXT-98-00834, Revision 1.1b.

Schnurr, N. M. et al., 1992. TRAC-PF1/MOD2 code manual - user's guide, NUREG/CR-5673.

Thurgood, M.J., Kelly, J.M., Guidotti, T.E., Kohrt, R.J., Crowell, K.R., 1983. "COBRA/TRAC - a thermal hydraulics code for transient analysis of nuclear reactor vessels and primary coolant systems," NUREG/CR3046.

Weaver, W.L., Tomlinson, E.T., Aumiller, D.L., 2000. "A Generic Semi-Implicit Coupling Methodology For Use In RELAP5-3D ${ }^{\mathcal{Q}}$, , Available as B-T-3321 from DOE Office of Scientific and Technical Information. 
2001 RELAP5 International Users Seminar

Sun Valley, Idaho

September 5-7, 2001

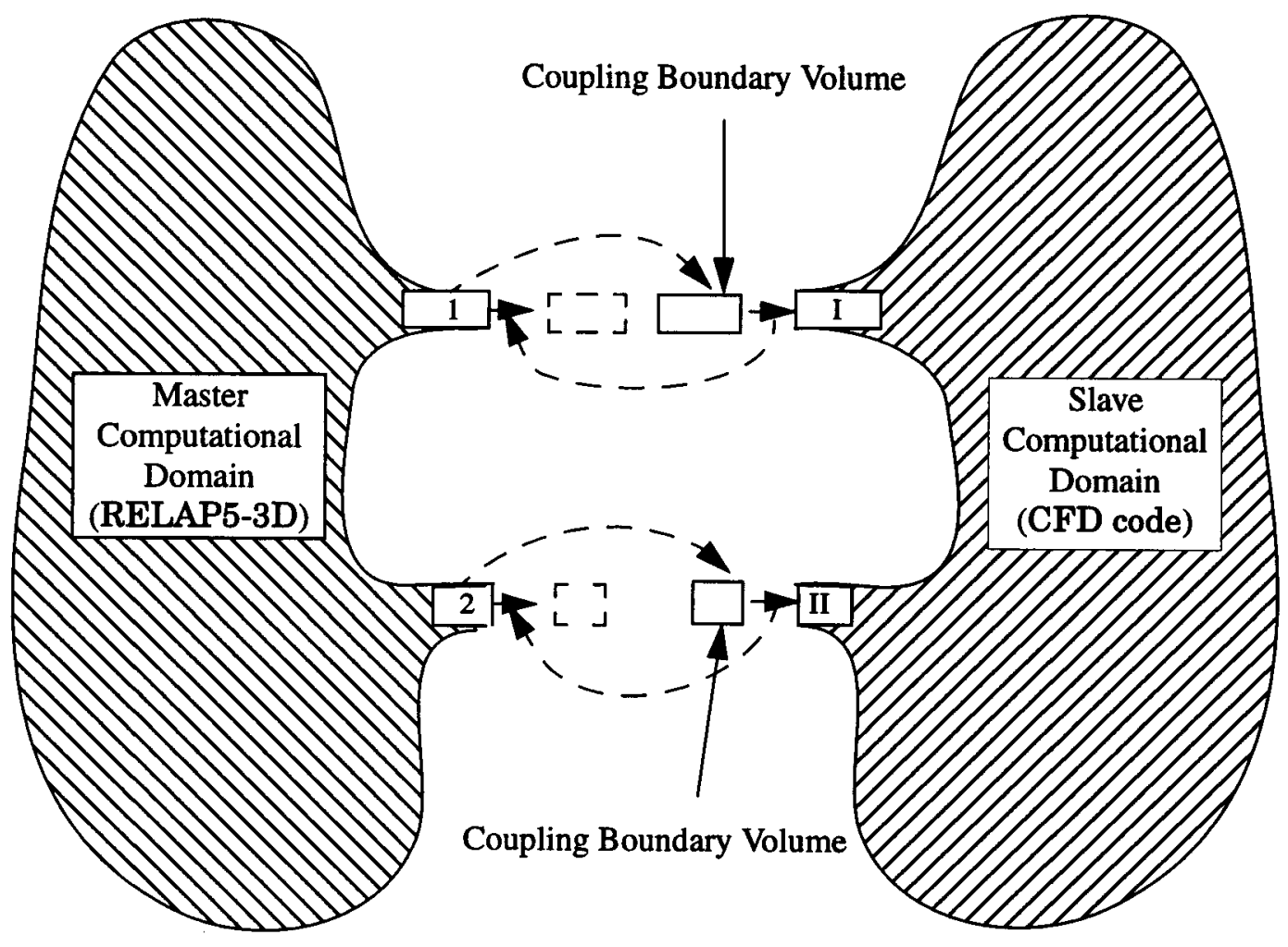

Figure 1: Schematic of Semi-Implicit Coupling Methodology

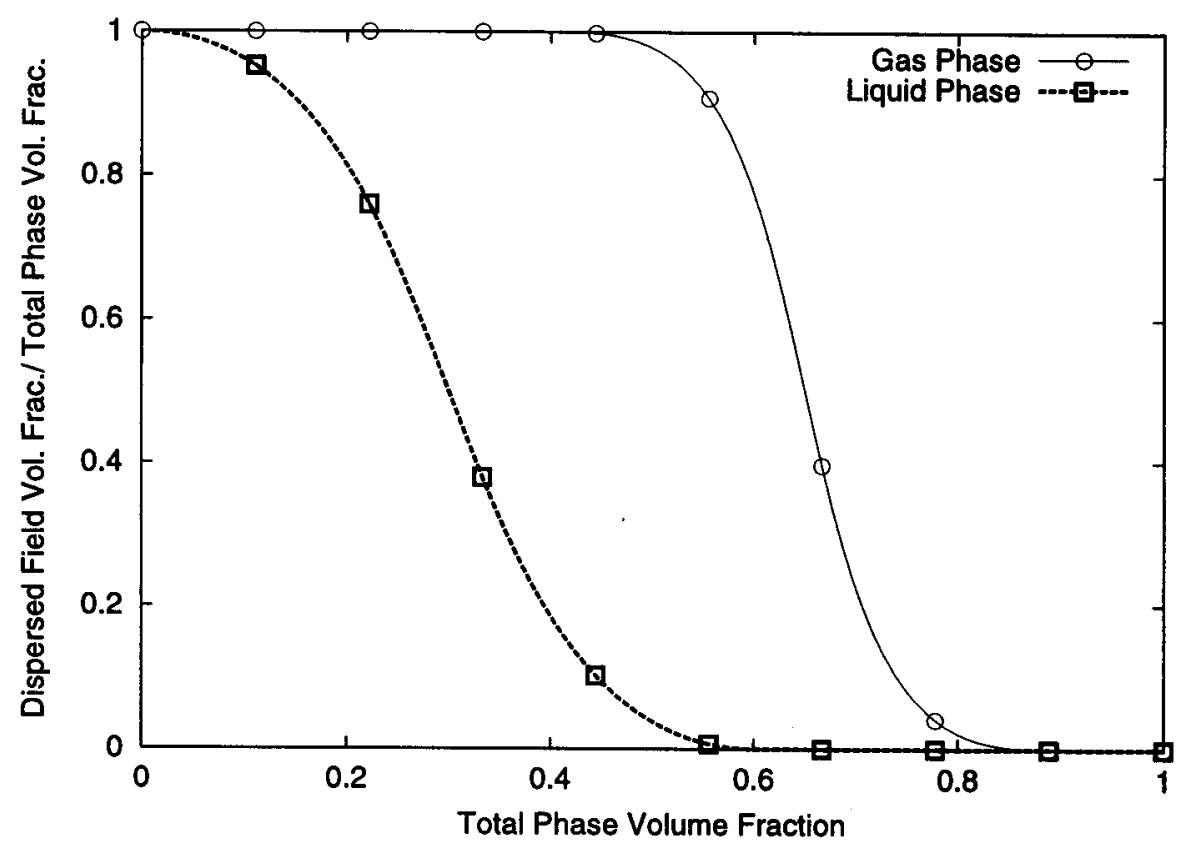

Figure 2: Data Creation for Gas and Liquid Phases 


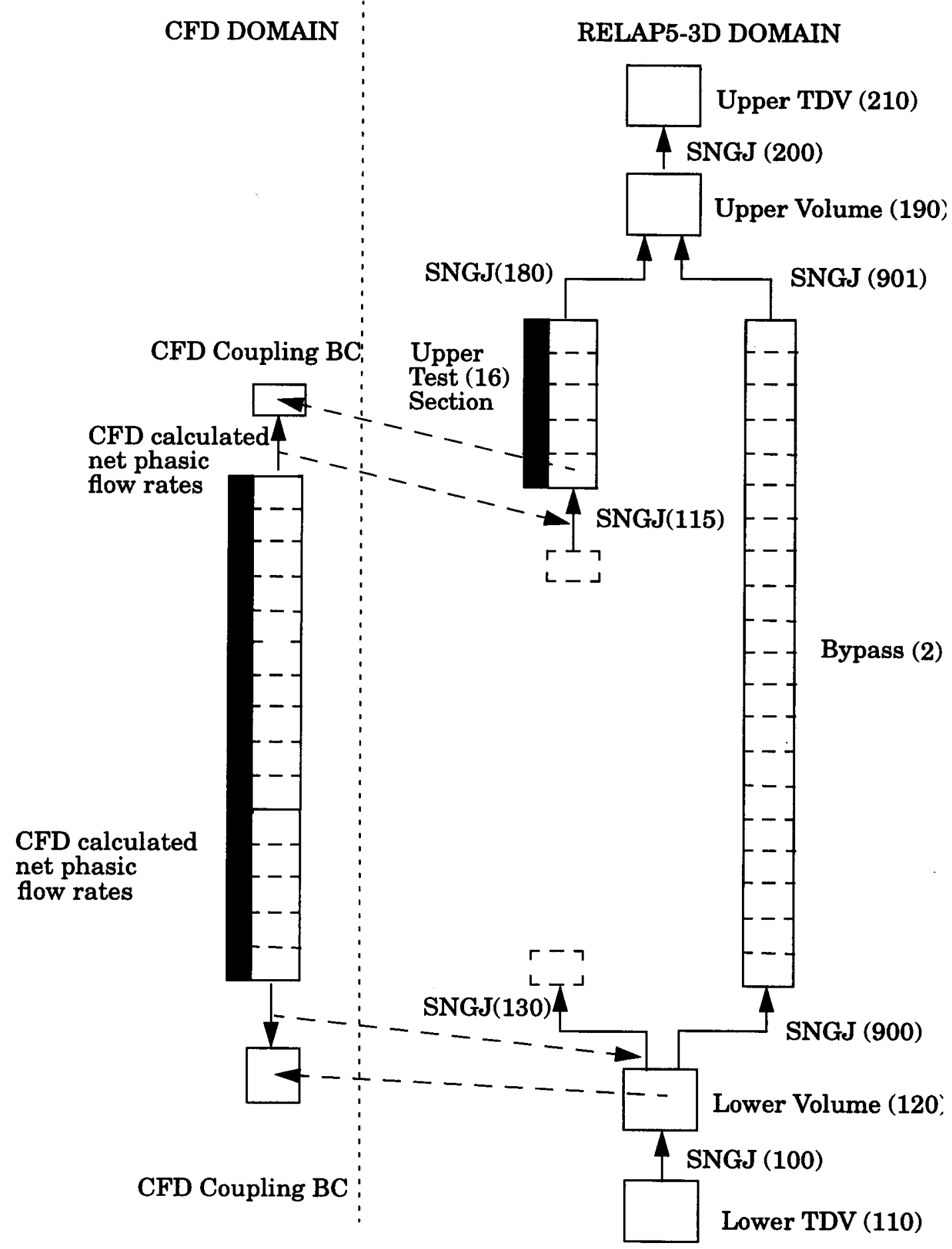

Figure 3: Schematic of the Coupled Representation of the Modified Christensen Experiment 
2001 RELAP5 International Users Seminar

Sun Valley, Idaho

September 5-7, 2001

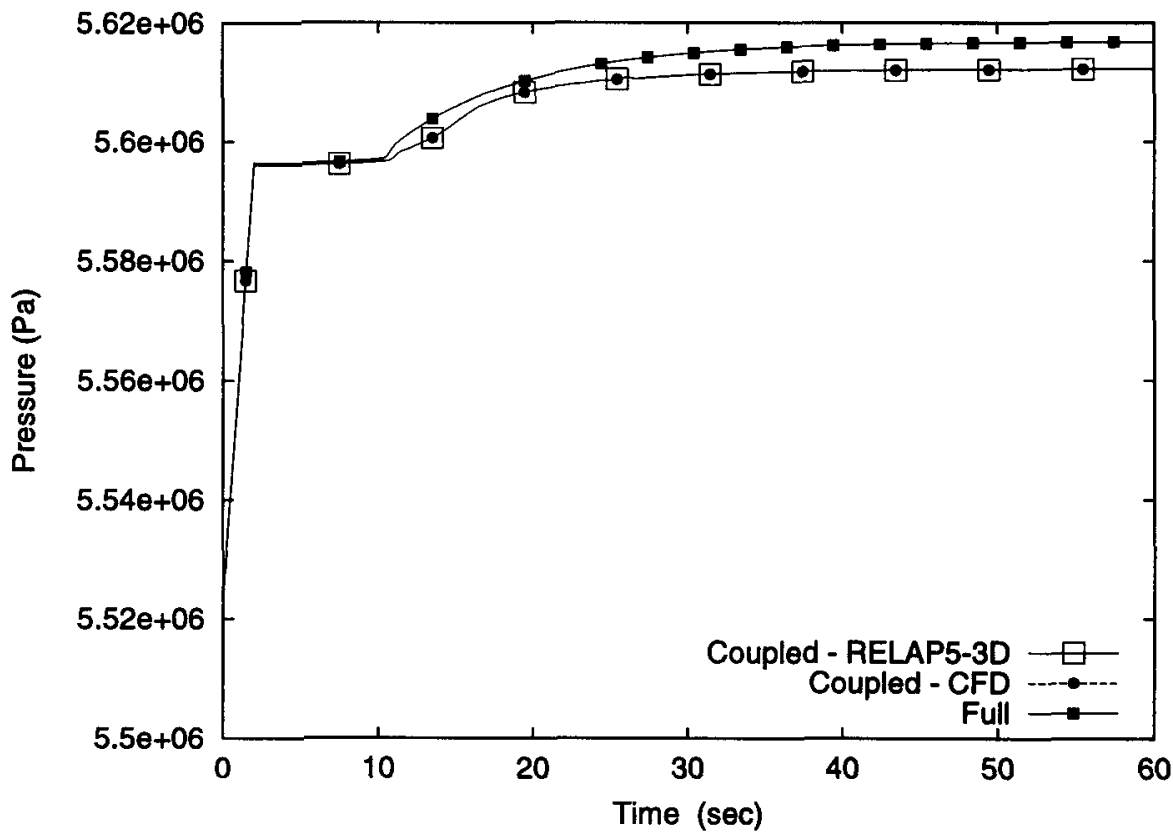

Figure 4: Comparison of Pressures in Lower Coupling Volume

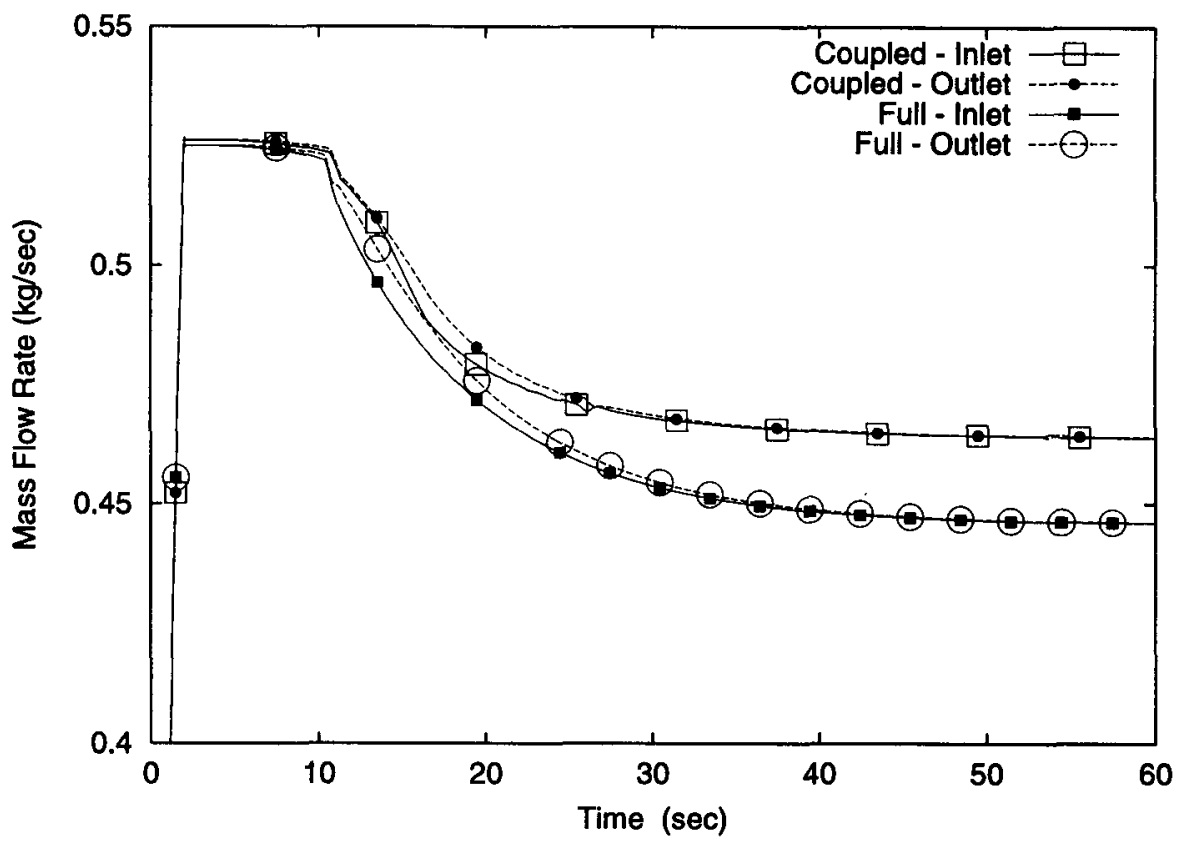

Figure 5: Comparison of Mass Flow Rates at Both Coupling Junctions 
2001 RELAP5 International Users Seminar

Sun Valley, Idaho

September 5-7, 2001

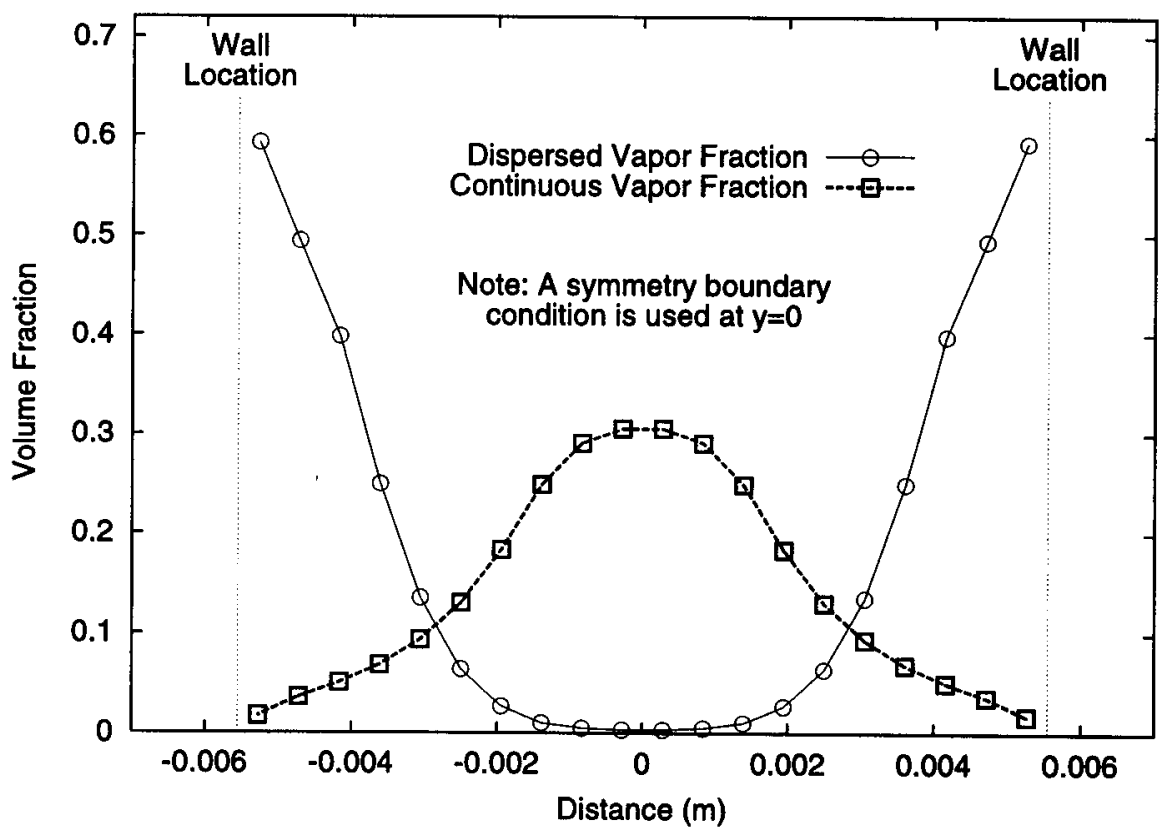

Figure 6: Calculated Void Fraction Profiles for Both Gas Fields in the CFD Domain at the Exit at 60 seconds

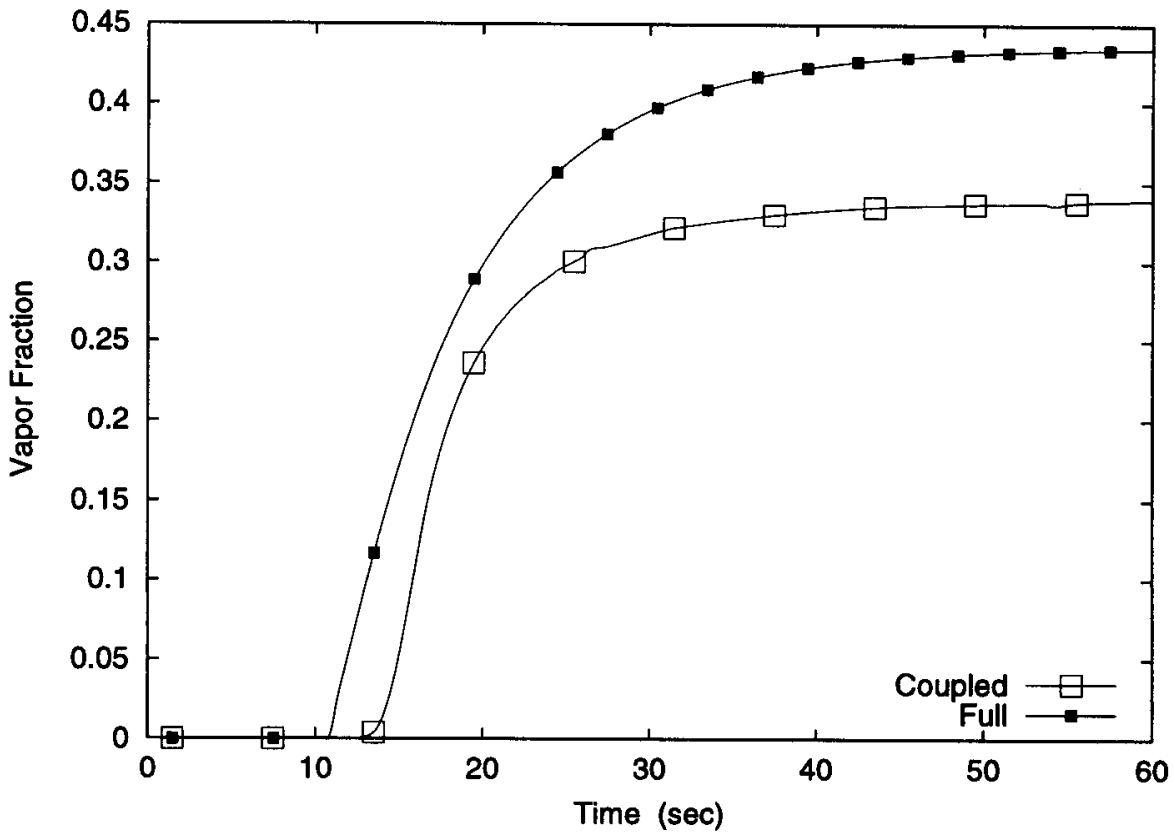

Figure 7: Comparison of Convected Void Fraction at the Upper Coupling Location 